\title{
La place de la communication dans la conception de systèmes à risques
}

Arlette Bouzon, Paris, L'Harmattan, 2004

Elizabeth Gardère

\section{OpenEdition}

\section{Journals}

Édition électronique

URL : http://journals.openedition.org/communicationorganisation/3264

DOI : 10.4000/communicationorganisation.3264

ISSN : 1775-3546

Éditeur

Presses universitaires de Bordeaux

Édition imprimée

Date de publication : 1 juin 2005

ISSN : 1168-5549

\section{Référence électronique}

Elizabeth Gardère, "La place de la communication dans la conception de systèmes à risques »,

Communication et organisation [En ligne], 27 | 2005, mis en ligne le 07 juin 2012, consulté le 21 septembre 2020. URL : http://journals.openedition.org/communicationorganisation/3264 ; DOI :

https://doi.org/10.4000/communicationorganisation.3264

Ce document a été généré automatiquement le 21 septembre 2020

(C) Presses universitaires de Bordeaux 


\section{La place de la communication dans la conception de systèmes à risques}

Arlette Bouzon, Paris, L'Harmattan, 2004

Elizabeth Gardère

1 Dans la complexité organisationnelle des projets, Arlette Bouzon ${ }^{1}$ choisit d'analyser les «systèmes techniques complexes » qui présentent de nombreux risques. La pertinence de cet objet de recherche s'appuie sur une enquête de terrain menée au sein d'équipes de projets spatiaux. L'ambition de l'auteur est de démontrer quelle est la place de la communication dans le processus de création collective de projets innovants à risques maîtrisés comme la conception d'avion, d'une centrale nucléaire, etc. Cependant, paradoxalement, les domaines de haute technologie (comme le secteur spatial), ne recouvrent pas que des produits innovants.

2 Ce type de projet s'inscrit dans une situation incertaine et évolutive. Et ce, d'autant plus que les acteurs sociaux sont soumis à des contraintes de temps, de délais et de ressources dans un contexte où les intérêts divergent parfois malgré l'interdépendance nécessaire de sous-ensembles inclus dans un système plus large. Tout processus de conception innovante se construit au fur et à mesure de l'action. Cela génère des fluctuations dans les objectifs et des incertitudes mais aussi une gestion de projet fondée sur une mosaïque de savoirs existants et de nouvelles expertises.

3 C'est dans un contexte d'économie globalisée que l'entreprise innovante s'affirme au niveau de la recherche. C'est pourquoi Arlette Bouzon évoque la réalité des pratiques quotidiennes des acteurs de la conception et de l'innovation dans l'organisation qui semble en décalage avec l'engouement prescriptif de certaines conceptions. Or ce qui différencie principalement la conception innovante de la conception routinière, c'est son organisation et son degré d'incertitude.

Dans un propos liminaire, Arlette Bouzon, convoque de nombreux auteurs tels que, entre autres, Akrich, Alter, Boutinet, Callon et Latour, Schumpeter, ou encore Veltz, dont les points communs sont d'inclure la communication dans leurs analyses ou d'établir des liens entre conception et innovation. Ainsi, au filtre de ces références ciblées, le propos d'Arlette Bouzon est étayé de diverses définitions de l'innovation et 
du processus de conception innovante, termes qui jalonneront sa recherche. Cette précaution terminologique donne la préférence aux définitions qui corroborent les logiques de projet où cohabitent l'acquisition de connaissances, la conception et la prise de décisions dans des représentations collectives. L'ouvrage est scindé en deux chapitres. Dans un premier temps, les contours conceptuels et théoriques de la conception innovante et de la maîtrise des risques sont précisés et mis en perspective avec les structures de projet. Cette orientation permet d'élargir l'approche aux risques encourus par l'entreprise dans ce type de démarche et d'envisager une maîtrise des risques adaptée à la conception innovante.

5 Par ailleurs, une seconde partie développe la problématique des représentations des acteurs dans la coopération des processus de coopération. La spécificité de ce travail de recherche par rapport aux autres ouvrages sur l'innovation est d'être fondé sur une enquête de terrain minutieuse et dense. Arlette Bouzon s'inscrit «dans la lignée des travaux d'inspiration interactionniste et ethnométhodologique ». Aussi, la démarche compréhensive adoptée est basée sur des entretiens approfondis, l'observation de situations et l'analyse de documents. A noter que l'auteur a dû faire face lors de son travail d'enquête, aux difficultés liées à la confidentialité du domaine aéronautique et spatial. Malgré cela, la richesse des résultats obtenus hors observation participante, et l'analyse qui en est faite, permettent une lecture critique de la place de la communication dans la conception de systèmes à risques.

Dans ses conclusions, Arlette Bouzon annonce ainsi que « la communication se révèle-telle omniprésente dans les processus de conception innovante à risques maîtrisés " même si cette dimension semble parfois oubliée dans un univers technique. Cette thèse est étayée par le point de vue selon lequel il n'y a pas d'innovation sans espace de liberté. Le décloisonnement hiérarchique au profit d'un engagement d'une pluralité d'acteurs et de compétences est une condition de réussite des processus de conception.

7 La coopération et la coordination sont donc au cœur de cette réflexion à lire pour mieux comprendre, notamment, la confrontation des théories relatives à l'interaction au travail avec l'observation des pratiques sur le terrain qui « conduit à reconnaître l'influence des représentations des acteurs sur leurs actions, durant l'activité de conception ». La cohérence entre les représentations et les divergences représentationnelles des acteurs sont alors autant de paramètres à prendre en compte par la communication dans la conception des systèmes à risques. Dans ces systèmes complexes et sensibles à un environnement incertain et variable, Arlette Bouzon donne donc à son lecteur un autre éclairage. C'est à la lumière de la communication organisationnelle qu'elle traite des questions des représentations collectives, de la gestion de la connaissance, de la circulation de l'information et de l'implantation de mécanismes de surveillance et de protection dans les systèmes à risques. 


\section{NOTES}

1. Professeur de Sciences de l'Information et de la Communication à l'Université Toulouse 3, membre du LERASS et animatrice du groupe d'études et de recherches en communication des organisations (Org\&Co) de la S.F.S.I.C.

\section{AUTEUR \\ ELIZABETH GARDÈRE}

205-207 